\title{
Cell free circulating tumor DNA in cerebrospinal fluid detects and monitors central nervous system involvement of B-cell lymphomas
}

\author{
Sabela Bobillo, ${ }^{1 *}$ Marta Crespo, ${ }^{1 *}$ Laura Escudero, ${ }^{2 *}$ Regina Mayor, ${ }^{2}$ \\ Priyanka Raheja, ${ }^{1}$ Cecilia Carpio, ${ }^{1}$ Carlota Rubio-Perez, ${ }^{2}$ \\ Bárbara Tazón-Vega, ${ }^{1}$ Carlos Palacio, ${ }^{1}$ Júlia Carabia, ${ }^{1}$ Isabel Jiménez, ${ }^{1}$ \\ Juan. C. Nieto, ${ }^{1}$ Julia Montoro, ${ }^{1}$ Francisco Martínez-Ricarte, ${ }^{3}$ Josep Castellví, ${ }^{4}$ \\ Marc Simó, ${ }^{5}$ Lluis Puigdefàbregas, ${ }^{1}$ Pau Abrisqueta, ${ }^{1}$ Francesc Bosch ${ }^{17}$ \\ and Joan Seoane ${ }^{2,6 \#}$
}

\begin{abstract}
${ }^{1}$ Laboratory of Experimental Hematology, Department of Hematology, Vall d'Hebron Institute of Oncology (VHIO), Vall d'Hebron University Hospital (HUVH), Universitat Autònoma de Barcelona, Department of Medicine; ${ }^{2}$ Translational Research Program, Vall d'Hebron Institute of Oncology (VHIO), Vall d'Hebron University Hospital (HUVH), Universitat Autònoma de Barcelona; ${ }^{3}$ Department of Neurosurgery, Universitat Autònoma de Barcelona, Hospital Vall d'Hebron; ${ }^{4}$ Department of Pathology, Vall d'Hebron University Hospital, Universitat Autònoma de Barcelona; ${ }^{5}$ Department of Nuclear Medicine, Vall d'Hebron University Hospital, Universitat Autònoma de Barcelona and 'Institució Catalana de Recerca i Estudis Avançats (ICREA), Barcelona, Spain and CIBERONC
\end{abstract}

${ }^{*} \mathrm{SB}, \mathrm{MC}$ and LE contributed equally as co-first authors.

"FB and JS contributed equally as co-senior authors.

\section{Perrata Storti Foundation}

Haematologica 2021

Volume 106(2):513-521

\section{ABSTRACT}

T The levels of cell free circulating tumor DNA (ctDNA) in plasma correlate with treatment response and outcome in systemic lymphomas. Notably, in brain tumors, the levels of ctDNA in the cerebrospinal fluid (CSF) are higher than in plasma. Nevertheless, their role in central nervous system (CNS) lymphomas remains elusive. We evaluated the CSF and plasma from 19 patients: 6 restricted CNS lymphomas, 1 systemic and CNS lymphoma, and 12 systemic lymphomas. We performed whole exome sequencing or targeted sequencing to identify somatic mutations of the primary tumor, then variant-specific droplet digital polymerase chain reaction was designed for each mutation. At time of enrollment, we found ctDNA in the CSF of all patients with restricted CNS lymphoma but not in patients with systemic lymphoma without CNS involvement. Conversely, plasma ctDNA was detected in only 2 out of 6 patients with restricted CNS lymphoma with lower variant allele frequencies than CSF ctDNA. Moreover, we detected CSF ctDNA in one patient with CNS lymphoma in complete remission and in one patient with systemic lymphoma, 3 and 8 months before CNS relapse was confirmed, indicating that CSF ctDNA might detect CNS relapse earlier than conventional methods. Finally, in two cases with CNS lymphoma, CSF ctDNA was still detected after treatment even though no tumoral cells were observed by flow cytometry (FC), indicating that CSF ctDNA detected residual disease better than FC. In conclusion, CSF ctDNA can detect CNS lesions better than plasma ctDNA and FC. In addition, CSF ctDNA predicted CNS relapse in CNS and systemic lymphomas.

\section{Introduction}

Central nervous system (CNS) involvement in B-cell malignancies is associated with dismal prognosis, especially in the relapse setting. ${ }^{1}$ Primary central nervous system lymphoma (PCNSL) is defined by the presence of CNS lymphoma in the absence of systemic disease. ${ }^{2}$ Conversely, secondary involvement of the CNS (SCNSL) is characterized by CNS infiltration with concomitant or previous history of systemic lymphoma. ${ }^{3}$ The diagnosis of CNS lymphoma is commonly based on cra-

\section{Correspondence: \\ JOAN SEOANE \\ jseoane@vhio.net}

FRANCESC BOSCH

fbosch@vhio.net

Received: October 22, 2019.

Accepted: February 18, 2020.

Pre-published: February 20, 2020.

https://doi.org/10.3324/haematol.2019.241208

(C)2021 Ferrata Storti Foundation

Material published in Haematologica is covered by copyright. All rights are reserved to the Ferrata Storti Foundation. Use of published material is allowed under the following terms and conditions:

https://creativecommons.org/licenses/by-nc/4.0/legalcode. Copies of published material are allowed for personal or internal use. Sharing published material for non-commercial purposes is subject to the following conditions: https://creativecommons. org//licenses/by-nc/4.0/legalcode, sect. 3. Reproducing and sharing published material for commercial purposes is not allowed without permission in writing from the publisher. 
nial magnetic resonance imaging (MRI) along with brain excisional biopsy or analysis of cerebrospinal fluid (CSF) or vitreous fluid. ${ }^{4}$ In some cases, biopsies can be challenging owing to the difficulty in accessing the tumor and the inherent risks associated with cranial surgery.

Patients with aggressive systemic lymphomas and risk factors for CNS relapse are routinely monitored by cytology and flow cytometry (FC) analysis of CSF. Cytology is highly specific but has a very limited sensitivity, showing up to $40 \%$ false negatives. In contrast, FC is more sensitive, detecting malignant cells in up to $5-15 \%$ of patients with negative cytology. ${ }^{5,6}$ However, FC still has some limitations, and in a relevant fraction of patients, recurrence in the CNS is found shortly after a negative result by FC, suggesting that tumor cells were undetected.

Several studies have demonstrated that cell free circulating tumor DNA (ctDNA) can be detected in plasma from patients with B-cell lymphomas and correlates with metabolic tumor volume and outcome., ${ }^{7,8}$ Due to the difficulties in the diagnosis of brain tumors, there is growing interest in the analysis of ctDNA in plasma and CSF and their potential utility in the management of these patients. Thus, we and others have explored the analysis of CSF in solid brain tumors as a better source of ctDNA compared with plasma. ${ }^{9-12}$ As per B-cell lymphomas, only a minority of patients with restricted CNS lymphomas present detectable ctDNA in plasma ${ }^{13,14}$ and only a few studies of ctDNA in CSF have been performed so far. ${ }^{15-19}$ In this regard, the MYD88 L265P mutation has been identified in the CSF ctDNA from some patients with PCNSL and lymphoplasmacytic lymphoma with CNS involvement. ${ }^{17-19}$ More recently, a different study using a next-generation sequencing (NGS)-based assay, detected at least one tumor-derived genetic alteration in the CSF from eight patients with relapsed CNS lymphoma and demonstrated that CSF ctDNA levels correlated with treatment response. ${ }^{15}$

Taken together, these studies demonstrate that CSF ctDNA could be detected in patients with CNS lymphomas and could be useful to monitor the disease, but a thorough analysis of its relevance on the diagnosis and monitoring of the disease is still needed.

Here, we conducted a study to explore whether the analysis of ctDNA in the CSF and plasma could be useful to complement the diagnosis and molecular profile of tumors, as well as to monitor treatment response in CNS lymphomas. In addition, we evaluated whether the presence of CSF ctDNA in patients with systemic lymphoma could be more sensitive to detect CNS malignancies than CSF standard analyses and plasma ctDNA.

\section{Methods}

\section{Patients and samples}

Nineteen patients diagnosed with the following conditions were included: restricted CNS lymphomas, $n=6$; PCNSL, $n=1$; SCNSL, $\mathrm{n}=5$; systemic lymphoma with concomitant CNS involvement, $\mathrm{n}=1$; and systemic lymphoma without CNS disease but risk factors for CNS relapse, $\mathrm{n}=12$. Risk factors for CNS relapse were defined as: diffuse large B-cell lymphoma (DLBCL) with testis, kidney or adrenal involvement; DLBCL with involvement of two or more extranodal sites along with high lactate dehydrogenase; high-grade B-cell lymphoma (HGBCL) with MYC and/or BCL2 rearrangements or Burkitt lymphoma. All patients were diagnosed and treated at Vall d'Hebron University Hospital (Barcelona,
Spain). Diagnosis of CNS disease was established by MRI imaging and tumor biopsy in cases with parenchymal involvement, or by FC and cytology in cases with leptomeningeal disease.

A written informed consent was obtained from all individuals in accordance with the Declaration of Helsinki and the study was approved by the local clinical research ethics committee. As part of standard of care practice, in patients with systemic lymphoma and risk factors for CNS relapse, prophylactic intrathecal (IT) methotrexate (MTX) was administered every 3 weeks together with systemic chemotherapy. In addition, in cases with leptomeningeal disease, IT chemotherapy was administrated every 3-4 days until malignant cells were not detected by FC. CSF (1-2 $\mathrm{mL})$ and plasma were collected before treatment in all patients and sequentially in patients who received IT chemotherapy as part of routine practice. Cytology and FC were performed in all CSF samples. Two out of 7 patients with CNS lymphoma and 3 out of 12 with systemic lymphoma received steroids before the baseline CSF sample. Evaluation of response was assessed at the end of treatment and/or on suspicion of disease progression by using MRI imaging in cases with CNS parenchymal involvement and by CSF analysis (FC and cytology) in patients with leptomeningeal disease. Positron emission tomography/computed tomography (PET/CT) was used to assess response at the end of treatment in patients with systemic lymphoma.

\section{Sample collection and DNA extraction}

The median volume of plasma and CSF obtained was $2.5 \mathrm{~mL}$ (range: $1-6$ ) and $1 \mathrm{~mL}$ (range: $0.3-2$ ), respectively. Peripheral blood was collected in tubes containing K2EDTA (Vacutainer) and centrifuged at $1600 \mathrm{xg}$ for 10 minutes (min). The plasma was then transferred to another tube that was further centrifuged at $3000 \mathrm{xg}$ for $5 \mathrm{~min}$. CSF samples were centrifuged at $3000 \mathrm{xg}$ for $5 \mathrm{~min}$. The supernatant was collected and CSF-derived and plasma-derived circulating cell free DNA (cfDNA) was extracted using the OIAamp Circulating Nucleid Acids kit and quantified using a fluorimeter. DNA was extracted from formalin-fixed paraffinembedded tumor samples using the OIAamp DNA FFPE tissue kit. Germline DNA was extracted from peripheral blood granulocytes using the Qiagen DNeasy Blood and Tissue kit.

\section{DNA sequencing and mutation genomic analysis}

We performed DNA sequencing of the tumors of 16 out of 19 patients: 11 of 16 were sequenced using a 300 gene targeted NGS panel (Vall d'Hebron Institute of Oncology, Genomics Facility) and in the remaining five, whole-exome DNA sequencing (WES) was performed in both the tumor and germline DNA. SureSelect Human All Exon V5 (Agilent Technologies) was used to perform whole exome enrichment for the Illumina paired-read sequencing platform HiSeq2500 with a read length of 2x100bp (Online Supplementary Appendix). The amount of DNA used for NGS panel and WES was $1 \mu \mathrm{g}$ and $0.4-4 \mu \mathrm{g}$, respectively. In 3 out of 19 patients (NHL1, NHL4 and NHL5), MYD88 L265P mutation was detected in the tumor by routine genomic analysis performed at diagnosis, therefore tumor DNA sequencing was not performed.

\section{Droplet digital polymerase chain reaction and quantification of circulating tumor-specific DNA}

Given the limited amount of cfDNA available, ddPCR was performed to determine the presence of ctDNA mutations in the CSF and plasma, ensuring sensitivity and precision. A set of driver mutations along with additional mutations not predicted as drivers by Cancer Genome Interpreter (CGI), but relevant according to expert knowledge, were selected for further validation by ddPCR.

Custom Taqman SNP genotyping assays for ddPCR were designed to specifically detect the selected point mutations and 
A

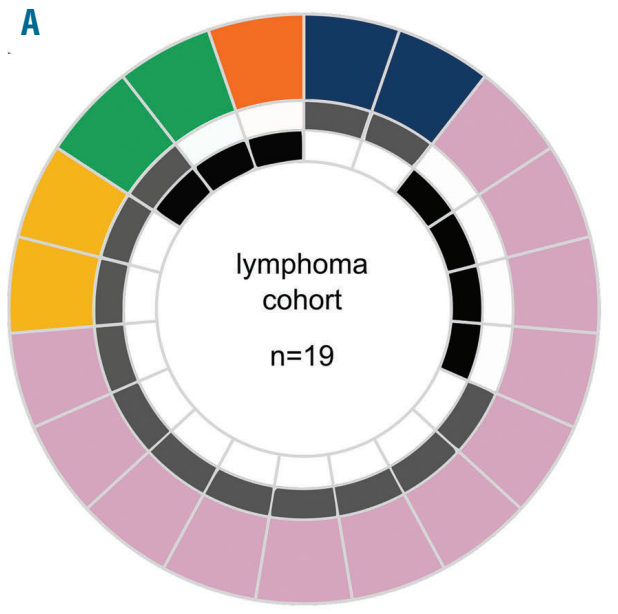
BL WM
DLBCL $\begin{aligned} & \text { CNS involvement } \\ & \text { systemic involvement } \\ & \text { HG BCL }\end{aligned}$

B

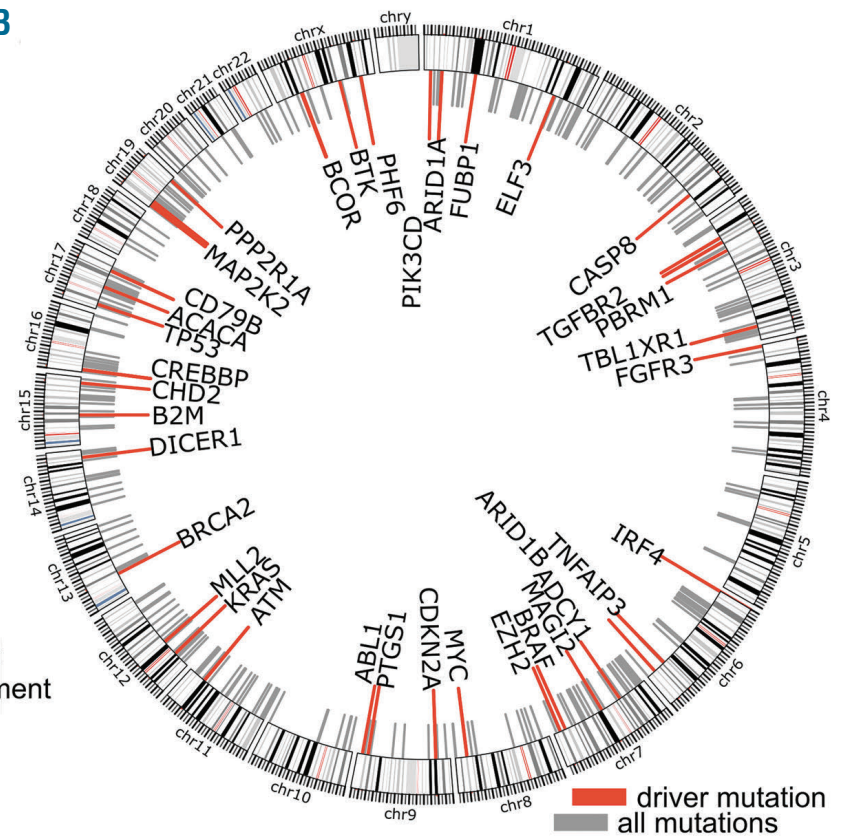
validated $O$ 0

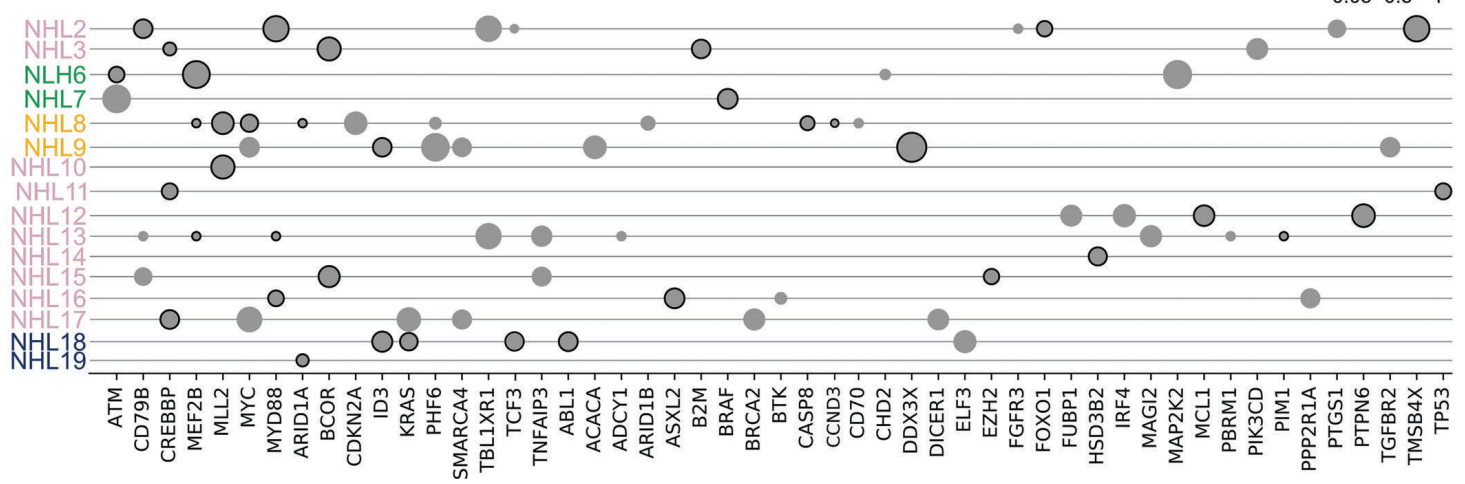

Figure 1. Genomic landscape of the cohort of study. (A) Pie chart representing the proportion of samples of each lymphoma type (see color legend). Two sectors in each sample depict whether the patient had systemic (gray) or central nervous system (CNS) restricted disease (black). (B) Circus plot representing all mutations identified across 16 lymphoma patients. A mutation was defined as driver according to Cancer Genome Interpreter (see Online Supplementary Appendix) colored in red (see Methods section). Genes with recurrent mutations have been highlighted in bold. (C) Bubble plot representing all driver mutations and droplet digital polymerase chain reaction (ddPCR) validated mutations identified in each patient. The bubble size represents the cancer allelic fraction (CAF) (see Online Supplementary Appendix) and the border has been highlighted in black if it has been found by ddPCR, in either the cerebrospinal fluid or plasma of each patient. NHL: non-Hodgkin lymphoma; DLBCL: diffuse large B-cell lymphoma; HGBCL: high-grade B-cell lymphoma; WM: Waldeström macroglobulinemia; MCL: mantle cell lymphoma; BL: Burkitt lymphoma.

the corresponding wild-type alleles. Genomic DNA from tumor tissues (10 ng), germline DNA from peripheral blood granulocytes (10 ng), plasma DNA, and CSF DNA (1-5 ng) were used for ddPCR analysis using the OX200 Droplet Digital PCR system according to manufacturer's protocols and the literature..$^{20}$

\section{Results}

Circulating tumor DNA in the cerebrospinal fluid of patients with central nervous system restricted B-cell lymphoma is more abundant than in plasma

Here, we sought to analyze the presence of ctDNA in the CSF and plasma from 19 patients with B-cell lymphomas with and without CNS disease at time of enrollment (Table 1). Six patients exhibited CNS restricted disease, one systemic and CNS disease, and 12 systemic disease with no
CNS involvement. CSF was obtained at the same time as plasma in all patients. WES or targeted sequencing of the tumors was performed to identify somatic mutations (Figure 1). Variant-specific ddPCR detecting 1-5 variants per patient was performed to detect ctDNA.

To demonstrate that the variant-specific ddPCR was highly specific, we performed MYD88 L265P ddPCR in ten CSF samples obtained from patients with hydrocephalus without a brain tumor $(\mathrm{n}=6)$, and from patients diagnosed with glioma $(n=3)$ and a cavernoma $(n=1)$. No mutant allele was found in any of the cases.

Analysis of the ctDNA in the CSF and plasma of the six CNS restricted lymphomas showed detectable ctDNA at high variant allele frequency (VAF) (ranging from 1\% to $95 \%$ ) in all cases. In contrast, ctDNA in plasma was only detected in 2 out of 6 cases at very low VAF (always $<5 \%$ ) (Table 1), highlighting that in CNS restricted lymphomas, 
Table 1. Patients' characteristics and tumor mutations at the time of enrolment and outcome.

\begin{tabular}{|c|c|c|c|c|c|c|c|c|c|c|c|c|c|}
\hline DD & Age & Histol & Dx & $\begin{array}{c}\text { Disease } \\
\text { status }\end{array}$ & $\begin{array}{c}\text { Systemic } \\
\text { inv }\end{array}$ & $\begin{array}{l}\text { CNS } \\
\text { inv }\end{array}$ & $\begin{array}{l}\text { CNS } \\
\text { (FC/C) }\end{array}$ & $\begin{array}{l}\text { Tumor } \\
\text { seq }\end{array}$ & $\begin{array}{l}\text { Tumor } \\
\text { mutations }\end{array}$ & $\begin{array}{l}\text { VAF } \\
\text { tumor }\end{array}$ & $\begin{array}{l}\text { VAF } \\
\text { CSF }\end{array}$ & $\begin{array}{c}\text { VAF } \\
\text { plasma }\end{array}$ & Outcome \\
\hline \multicolumn{14}{|c|}{ CNS lymphoma } \\
\hline NHL1 & 59 & DLBCL & PCNSL & New $\mathrm{dx}$ & No & $P$ & $-/-$ & - & MYD88 L265P & - & $48 \%$ & $\mathrm{~N}$ & CR \\
\hline \multirow[t]{4}{*}{ NHL2 } & \multirow[t]{4}{*}{60} & \multirow[t]{4}{*}{ DLBCL } & \multirow[t]{4}{*}{ SCNSL } & \multirow[t]{4}{*}{ Relapse } & \multirow[t]{4}{*}{ No } & \multirow[t]{4}{*}{ P, LM } & \multirow[t]{4}{*}{$+/-$} & \multirow[t]{4}{*}{ WES } & CD79B Y197D & $17 \%$ & $9 \%$ & - & \multirow[t]{4}{*}{ CR } \\
\hline & & & & & & & & & MYD88 L265P & $22 \%$ & $25 \%$ & - & \\
\hline & & & & & & & & & FOX01 D69A & $13 \%$ & - & - & \\
\hline & & & & & & & & & TMSB4A Q24P & $54 \%$ & $20 \%$ & - & \\
\hline \multirow[t]{3}{*}{ NHL3 } & \multirow[t]{3}{*}{53} & \multirow[t]{3}{*}{ DLBCL } & \multirow[t]{3}{*}{ SCNSL } & \multirow[t]{3}{*}{ Relapse } & \multirow[t]{3}{*}{ No } & \multirow[t]{3}{*}{ P, LM } & \multirow[t]{3}{*}{$+/+$} & \multirow[t]{3}{*}{ Panel } & B2M M1T & $41 \%$ & $\mathrm{~N}$ & $\mathrm{~N}$ & PD, died \\
\hline & & & & & & & & & BCOR N145S & $68 \%$ & $52 \%$ & $\mathrm{~N}$ & \\
\hline & & & & & & & & & CREBBP R1664C & $21 \%$ & $\mathrm{~N}$ & $\mathrm{~N}$ & \\
\hline NHL4 & 75 & WM & SCNSL & Relapse & No & LM & $+/+$ & - & MYD88 L265P & - & $33 \%$ & $\mathrm{~N}$ & $\mathrm{CR}$ \\
\hline NHL5 & 58 & DLBCL & SCNSL & Relapse & No & P, LM & $+/+$ & - & MYD88 L265P & $86 \%$ & $29 \%$ & $5 \%$ & CR, CNS relapse \\
\hline NHL6 & 73 & MCL & SCNSL & Relapse & No & LM & $+/+$ & Panel & ATM H2872L & $31 \%$ & $34 \%$ & $2 \%$ & PD, died \\
\hline & & & & & & & & & $M E F 2 B \mathrm{~K} 23 \mathrm{R}$ & $90 \%$ & $95 \%$ & $4 \%$ & \\
\hline CNS and & syst & mic lym & phoma & & & & & & & & & & \\
\hline NHL7 & 72 & MCL & SCNSL & Relapse & LN & LM & $+/+$ & Panel & $B R A F \mathrm{~N} 581 \mathrm{~S}$ & $44 \%$ & $\mathrm{~N}$ & $7 \%$ & PD, died \\
\hline Systemi & lym & homa & & & & & & & & & & & \\
\hline NHL8 & 50 & HGBCL & - & New dx & LN, & No & $-/-$ & WES & MYC Y27C & $28 \%$ & $\mathrm{~N}$ & $16 \%$ & CR \\
\hline & & & & & bone, & & & & ARID1A E1104 & $32 \%$ & $\mathrm{~N}$ & $20 \%$ & \\
\hline & & & & & intestine & & & & MEF2B D83G & $33 \%$ & $\mathrm{~N}$ & $18 \%$ & \\
\hline & & & & & & & & & MLL2 C533fs & $50 \%$ & $\mathrm{~N}$ & $31 \%$ & \\
\hline & & & & & & & & & CCND3 P284fs & $29 \%$ & $\mathrm{~N}$ & $16 \%$ & \\
\hline NHL9 & 38 & HGBCL & - & New dx & LN, & No & $-/-$ & WES & ID3 Y48* & $38 \%$ & $\mathrm{~N}$ & $12 \%$ & CR \\
\hline & & & & & intestine & & & & $D D X 3 X \mathrm{Q} 360 \mathrm{fs}$ & $98 \%$ & $\mathrm{~N}$ & $15 \%$ & \\
\hline NHL10 & 73 & DLBCL & - & New dx & LN, & No & $-/$ & Panel & MLL2 splicing & $60 \%$ & $\mathrm{~N}$ & $19 \%$ & CR \\
\hline & & & & & kidney & & & & MLL2 P3139A & $34 \%$ & $\mathrm{~N}$ & $12 \%$ & \\
\hline NHL11 & 72 & DLBCL & - & New dx & LN, lung, & No & $-/-$ & Panel & TP53 R273C & $32 \%$ & $\mathrm{~N}$ & $12 \%$ & CR \\
\hline & & & & & ileum & & & & CREBBP Y145C & $25 \%$ & $\mathrm{~N}$ & $27 \%$ & \\
\hline NHL12 & 86 & DLBCL & - & New dx & LN, breast & No & $-/-$ & Panel & MCL1 L186F & $31 \%$ & $\mathrm{~N}$ & $0.9 \%$ & CR \\
\hline & & & & & & & & & PTNPN6 T80fs & $26 \%$ & $\mathrm{~N}$ & $\mathrm{~N}$ & \\
\hline NHL13 & 77 & DLBCL & - & New dx & LN, & No & $-/-$ & WES & MYD88 L265P & $29 \%$ & $\mathrm{~N}$ & $5 \%$ & CR \\
\hline & & & & & testes & & & & PIM1 L2F & $27 \%$ & $\mathrm{~N}$ & $3 \%$ & \\
\hline & & & & & & & & & MEF2B E77K & $27 \%$ & $\mathrm{~N}$ & $5 \%$ & \\
\hline NHL14 & 62 & DLBCL & - & New dx & Teste & No & $-/-$ & Panel & HSD3B2 L108W & $22 \%$ & $\mathrm{~N}$ & $\mathrm{~N}$ & CR \\
\hline & & & & & & & & & MYD88 L265P & $31 \%$ & $\mathrm{~N}$ & $\mathrm{~N}$ & \\
\hline NHL15 & 75 & DLBCL & - & New dx & LN, testes, & No & $-/$ & Panel & BCOR S1517fs & $63 \%$ & $\mathrm{~N}$ & $\mathrm{~N}$ & CR \\
\hline & & & & & liver, adrenal & & & & EZH2 P557L & $34 \%$ & $\mathrm{~N}$ & $\mathrm{~N}$ & \\
\hline $\mathrm{NHL}^{\dagger} 6^{\dagger}$ & 57 & DLBCL & - & New dx & LN, sinus, & No & $-/-$ & Panel & MYD88 V217F & $28 \%$ & $\mathrm{~N}$ & $\mathrm{~N}$ & CR, CNS relapse \\
\hline & & & & & bones & & & & ASXL2 K927R & $42 \%$ & $\mathrm{~N}$ & $\mathrm{~N}$ & \\
\hline NHL17 & 58 & HGBCL & - & New dx & LN & No & $-1-$ & Panel & CREBBP S1436I & $50 \%$ & $\mathrm{~N}$ & $16 \%$ & CR, CNS relapse \\
\hline NHL1 $8^{\dagger}$ & 60 & BL & - & New dx & $\mathrm{LN}$, & No & $-/-$ & WES & ID3 L54V & $37 \%$ & $\mathrm{~N}$ & $\mathrm{~N}$ & CR \\
\hline & & & & & intestine & & & & $K R A S \mathrm{G} 12 \mathrm{~V}$ & $34 \%$ & $\mathrm{~N}$ & $1 \%$ & \\
\hline & & & & & & & & & TCF3 D561E & $42 \%$ & $\mathrm{~N}$ & $\mathrm{~N}$ & \\
\hline & & & & & & & & & ABL1 G321R & $43 \%$ & $\mathrm{~N}$ & $0.5 \%$ & \\
\hline NHL19 ${ }^{\dagger}$ & 65 & BL & - & New dx & LN, bone & No & - & Panel & ARIDIA del & $9 \%$ & $\mathrm{~N}$ & $1 \%$ & CR \\
\hline
\end{tabular}

BL: Burkitt lymphoma; C: cytology; CNS: central nervous system; CR: complete response; CSF: cerebrospinal fluid; DLBCL: diffuse large B-cell lymphoma; Dx: diagnosis; FC: flow cytometry; HGBCL: high-grade B-cell lymphoma; Histol: histology; ID: patient identification number; inv: involvement; LM: leptomeningeal; LN: lymph nodes; MCL: mantle cell lymphoma; N: not detectable; Neg: negative; NHL: non-Hodgkin lymphoma; P: parenchyma; PCNSL: primary central nervous system lymphoma; PD: progressive disease; Pos: positive; SCNSL: secondary central nervous system lymphoma; seq: sequencing; VAF: variant allele frequency;WES: whole exome sequencing;WM:Waldeström macroglobulinemia. 'Plasma samples were collected after administration of systemic chemotherapy. 
A

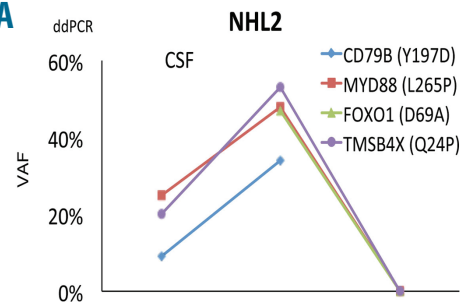

B

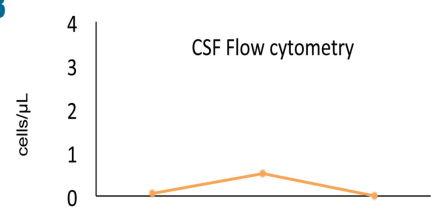

C
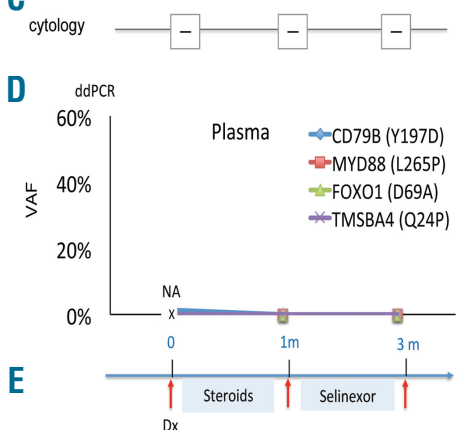

G $\quad$ ddPCR

NHL8

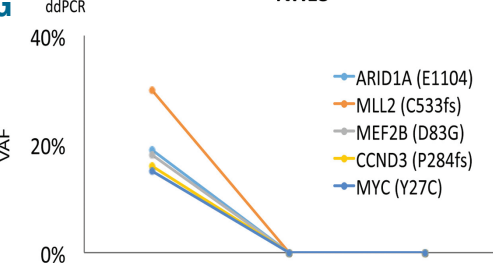

H
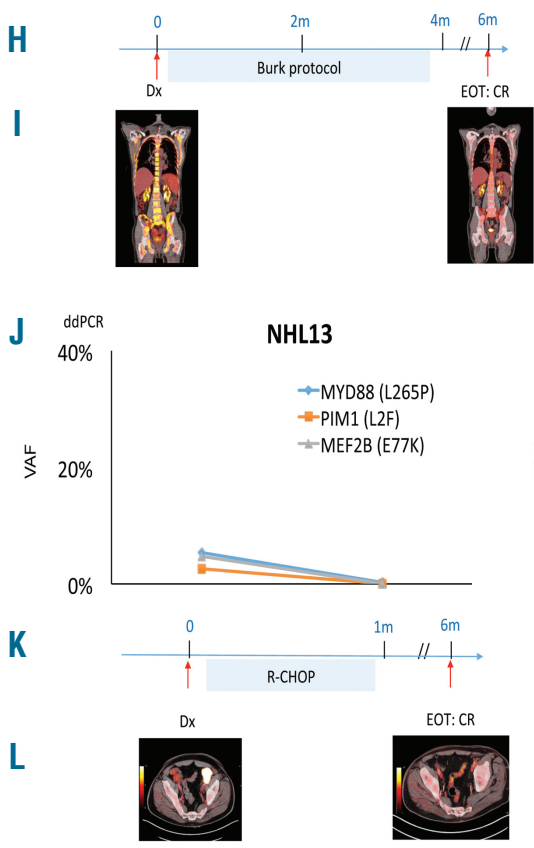
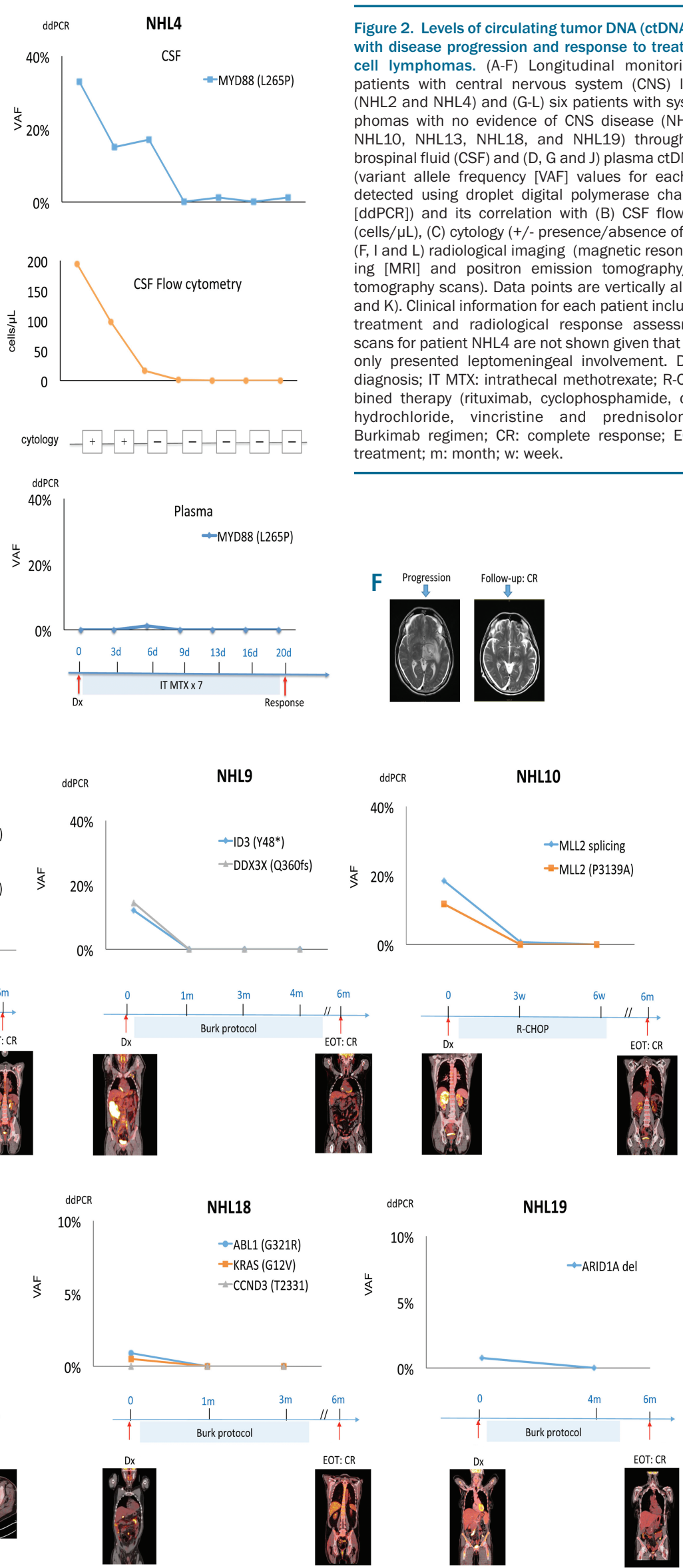
A
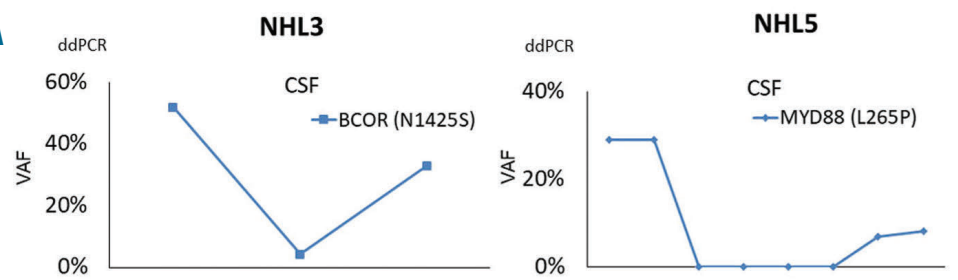

B
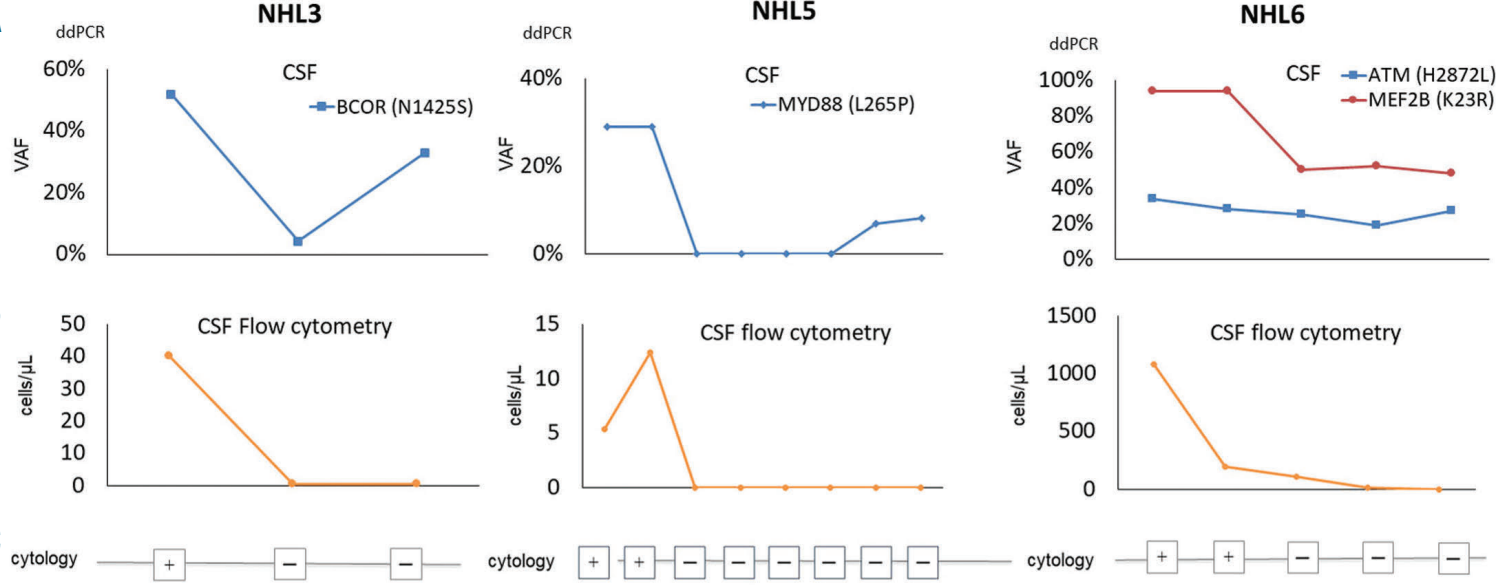

C
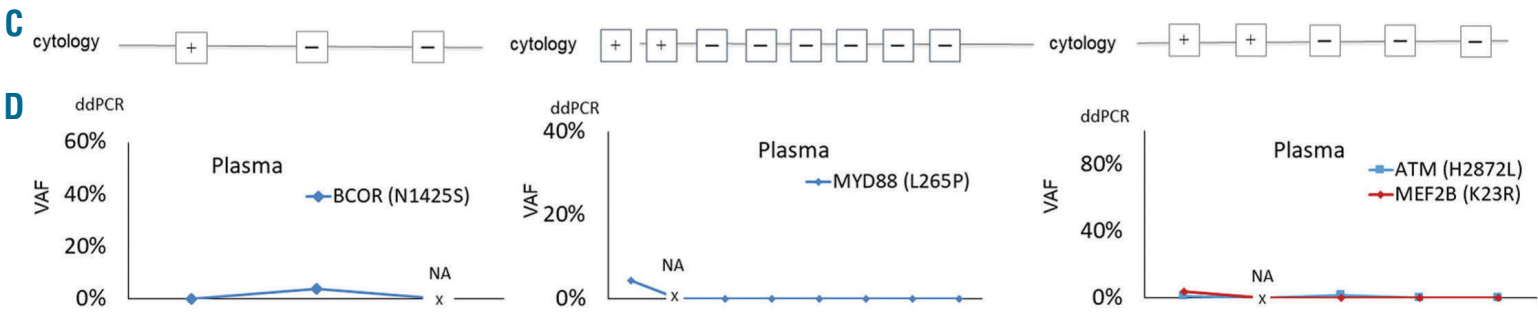

E
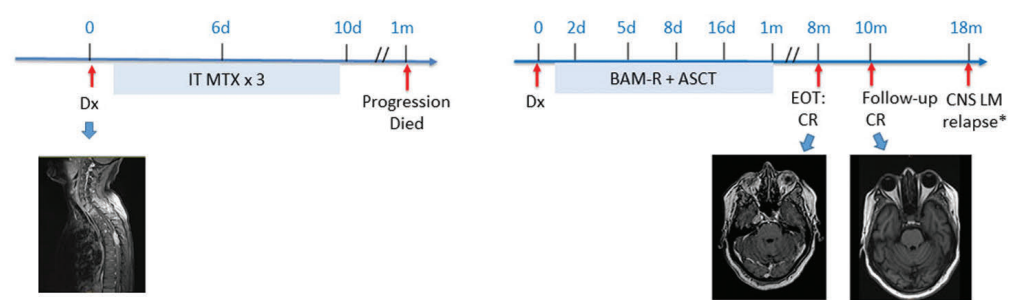

Figure 3. Cerebrospinal fluid (CSF) circulating tumor DNA (ctDNA) is more sensitive than flow cytometry (FC) to detect central nervous system (CNS) relapse and residual disease in CNS-restricted lymphomas. Longitudinal monitoring of three patients with CNS-restricted lymphomas (NHL3, NHL5 and NHL6) through (A) CSF and (D) plasma ctDNA analysis (variant allele frequency [VAF] values for each mutation obtained by droplet digital polymerase chain reaction [ddPCR]) and its correlation with (B) CSF flow cytometry (cells/ $\mu \mathrm{L})$, (C) cytology (+/- presence/absence of cells). (E) Type of treatment and response assessment, (F) radiological imaging (MRI). Data points are vertically aligned. MRI scans for patient NHL6 and NHL5 are not shown given that the patient only presented leptomeningeal involvement at diagnosis. Dx: time of diagnosis; IT MTX: intrathecal methotrexate; BAM-R: combined therapy (rituximab, carmustine, cytarabine and methotrexate); ASCT: autologous stem cell transplant; CR: complete response; LM: leptomeningeal; EOT: end of treatment; d: day; m: month; NA: not available. *CSF sample was not available for ctDNA analyses.

ctDNA is better identified in the CSF than in plasma. Interestingly, these two cases with restricted CNS lymphoma in which plasma ctDNA was detected had a previous history of systemic disease. This finding might reveal a systemic nature of some restricted SCNSL and deserves further investigation in larger studies.

We then analyzed 12 patients with systemic lymphoma without CNS involvement but with high risk for CNS relapse. In these cases, lumbar puncture was performed to rule out CNS infiltration and to administer IT MTX. As opposed to cases with CNS involvement, no ctDNA was detected in the CSF while it was detected at high VAF in the plasma of the majority of cases (Table 1). Finally, the patient with systemic and concomitant CNS disease had ctDNA in plasma but not in the CSF, suggesting that some CNS lesions cannot be captured through CSF ctDNA.

\section{Central nervous system circulating tumor DNA exhibits higher sensitivity than flow cytometry in detecting central nervous system lesions}

We next correlated the detection of ctDNA with the identification of malignant cells by conventional tests in the same CSF samples at time of enrollment. Cytology detected the presence of malignant cells in 4 out of 6 CNS restricted lymphomas. In these four cases the FC analysis also detect- ed lymphoma cells. In one case (NHL2), cytology was normal but a small amount of tumor cells was observed by FC (0.06 cell/ $/ \mathrm{L})$. Notably, all six cases exhibited CSF ctDNA. Importantly, in a patient with newly diagnosed PCNSL (NHL1) we could not detect lymphoma cells by FC or cytology; however, ctDNA was abundantly found in the CSF (Table 1). These results indicate that $\mathrm{ctDNA}$ analysis of the CSF can improve the detection of CNS lymphoma by conventional techniques.

\section{Cerebrospinal fluid circulating tumor DNA can be used to monitor central nervous system tumor burden and response to treatment}

To address whether the amount of CSF ctDNA could be useful to monitor tumor burden in CNS lymphomas, we analyzed sequential concomitant samples of CSF and plasma. CSF was obtained as part of the standard procedures before each administration of IT MTX or on suspicion of relapse. Tumor burden was evaluated by MRI and/or FC depending on the lymphoma location. We observed that CSF ctDNA levels correlated with disease response and progression (Figure 2). In addition, tumor burden measured by MRI and FC was concordant with CSF ctDNA levels in two patients with CNS lymphoma (Figure 2). Importantly, plasma ctDNA levels remained constantly low (VAF $<6 \%$ ) 


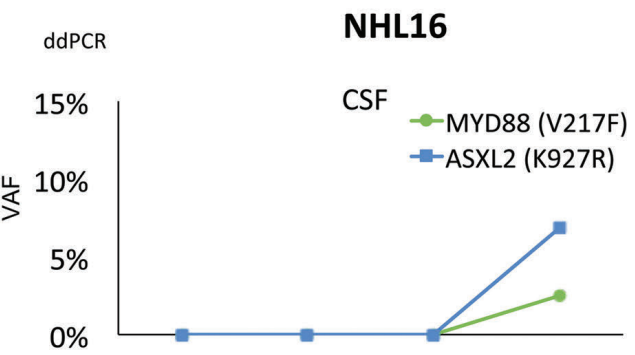

B

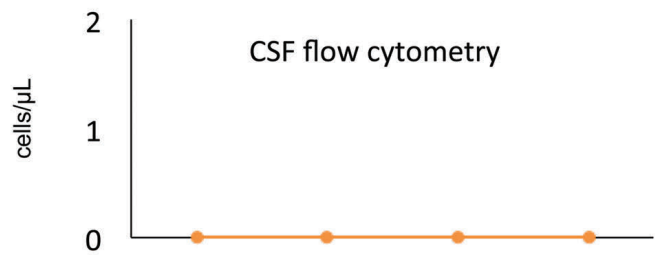

C

D

E
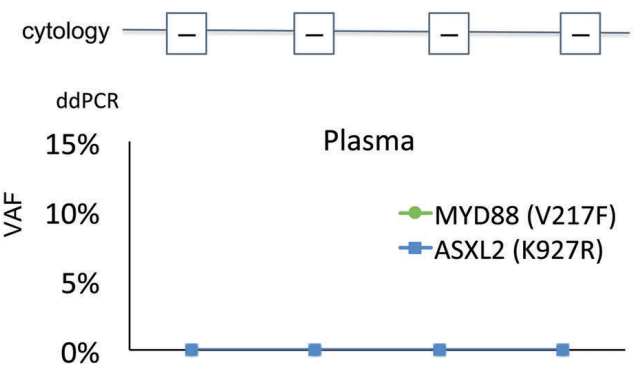
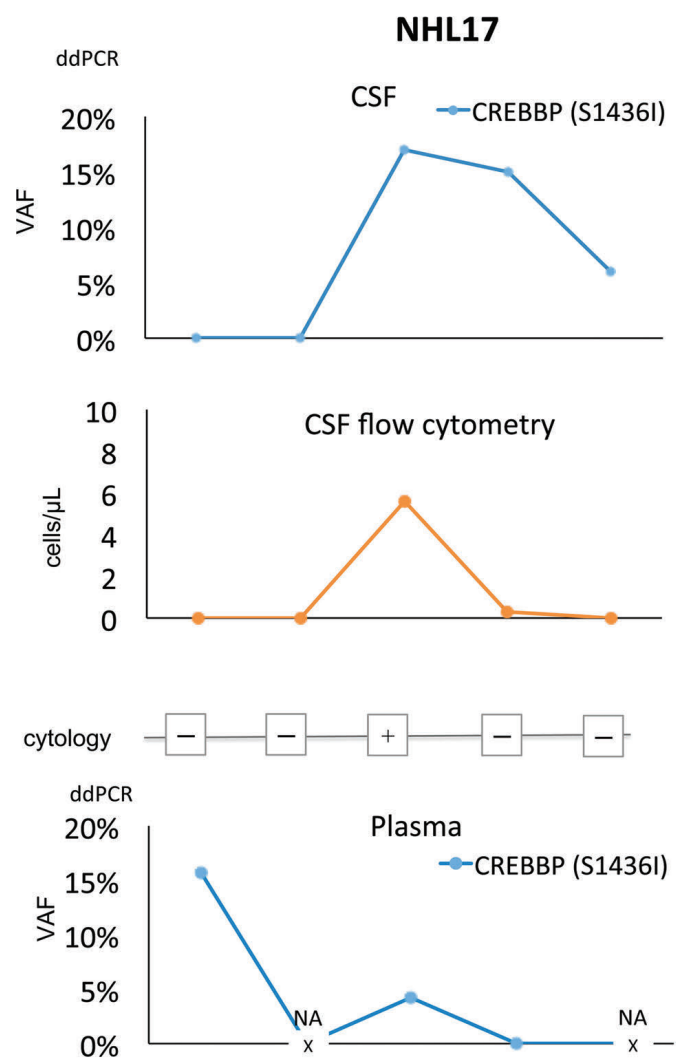

F
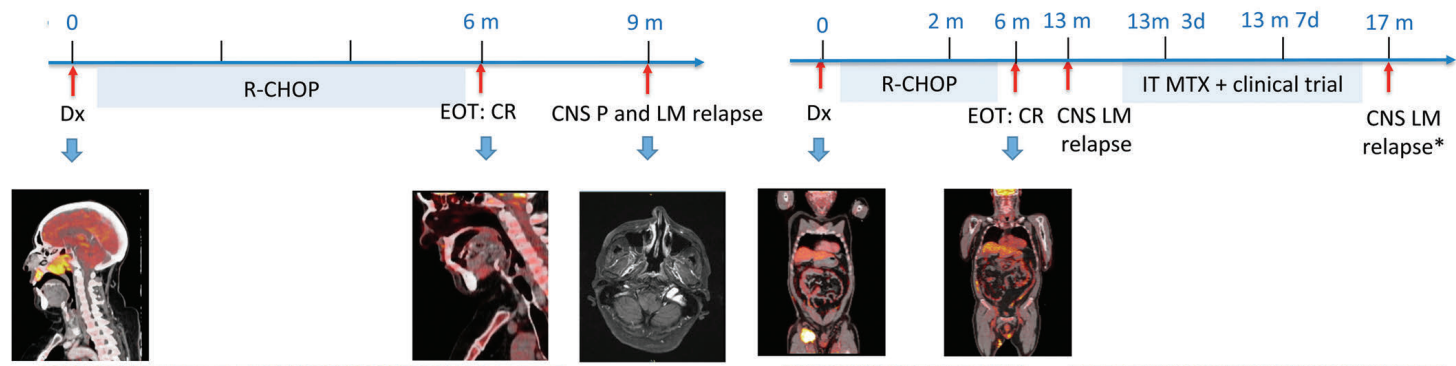

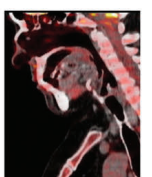

Systemic lymphoma

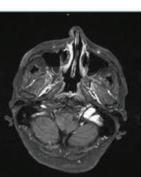

(a)

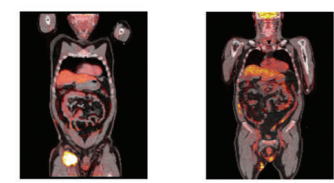

Systemic lymphoma
CNS lymphoma

Figure 4. Cerebrospinal fluid (CSF) circulating tumor DNA (ctDNA) detects central nervous system (CNS) involvement and residual disease in patients with systemic lymphoma and is more sensitive than conventional tests. Longitudinal analyses of (A) CSF and (D) plasma ctDNA (VAF values for each mutation detected using ddPCR) and correlation with (B) CSF FC (cells/ $u$ L), (C) cytology (+/- presence/absence of cells), and (E) type of treatment and radiological response assessment. ( $\mathrm{F}$ ) Radiological imaging (magnetic resonance imaging [MRI] and positron emission tomography/computed tomography scans) in two patients with systemic lymphoma (NHL16 and NHL17) that present CNS relapse. Data points are vertically aligned. MRI scans for patient NHL17 are not shown given that the patient only presented leptomeningeal involvement. Dx: time of diagnosis; R-CHOP: combined therapy (rituximab, cyclophosphamide, doxorubicin, vincristine and prednisone); IT MTX: intrathecal methotrexate; CR: complete response; P: parenchymal; LM: leptomeningeal; EOT: end of treatment; m: month; d: days; NA: not available. *CSF sample was not available for ctDNA analyses

in sequential samples and did not correlate with the disease status, underlining the fact that plasma ctDNA is not useful to monitor the activity of CNS restricted lymphomas (Figure 2).

Six patients with systemic lymphoma without CNS involvement had sequential plasma samples collected at the same time as CSF. We observed that plasma ctDNA became undetectable after the first cycle of treatment in all cases, in agreement with PET/CT results (Figure 2), suggesting that plasma ctDNA is a good tool to monitor response to treatment in these patients.

Cerebrospinal fluid circulating tumor DNA can identify central nervous system relapse and residual central nervous system disease better than flow cytometry

We then addressed whether the presence of ctDNA in the CSF could be more sensitive than conventional CSF analyses to monitor CNS lymphoma and detect CNS relapse.

In two cases of CNS lymphoma (NHL3 and NHL5), we observed that CSF ctDNA levels increased earlier than the detection of cells by FC. In NHL3, after the first dose of IT MTX, despite the fact that the patient did not exhibit a complete neurological response, we observed a sharp decrease in CSF ctDNA levels concomitant with a decrease in the tumor cells by FC. However, after three doses of IT MTX, although FC was negative, a clear ctDNA increase was observed. The patient died 1 month later due to progression (Figure 3). In NHL5, CSF ctDNA levels also decreased with response to treatment and remained undetectable after complete remission. However, CSF ctDNA reappeared in two CSF samples collected 2 and 4 months 
after treatment due to the presence of minimal neurological symptoms, when brain-spinal MRI and CSF FC did not show any evidence of disease; 8 months later CNS relapse was confirmed (Figure 3). Taken together, our results indicate that ctDNA can be a more sensitive tool than FC to monitor relapse of CNS lymphomas.

In one additional case of CNS lymphoma (NHL6) treatment with IT MTX decreased the number of cells in the CSF till they were no longer detectable by FC. However, CSF ctDNA was still detectable. Notably, this patient relapsed shortly after the analysis (Figure 3 ). In a similar way, we found persistence of ctDNA in the CSF from case NHL17 after treatment for CNS relapse, despite the fact that FC did not detect malignant cells. Four months later, the patient relapsed. (Figure 4). These results point out that the detection of CSF ctDNA may help to identify patients with residual disease who are likely to relapse.

A subset of patients with systemic lymphoma presents high risk of CNS relapse, which frequently occurs during treatment or shortly thereafter. To address whether the presence of CSF ctDNA could be more sensitive than FC to detect CNS disease, we analyzed sequential CSF samples from eight patients with systemic lymphoma obtained while receiving prophylactic IT MTX. Two out of 8 patients relapsed in the CNS during the time of study (Figure 4). In one case (NHL17), we found CSF ctDNA at the same moment in which FC showed a positive result. However, we could not detect ctDNA in the CSF samples collected 9 and 7 months before CNS progression. Notably, in case NHL16, the detection of ctDNA in the CSF with a VAF of $7 \%$ antedated by 3 months the CNS relapse. Importantly, at the time of ctDNA detection, the patient was asymptomatic and CSF FC was normal (Figure 4). This case points out that CSF ctDNA could detect CNS involvement in systemic lymphomas earlier than standard procedures. Finally, in the remaining six patients with systemic lymphoma and sequential CSF samples, ctDNA was not detected and, with a median follow-up of 18 months (range: 12-28 months), these patients did not develop CNS disease.

\section{Discussion}

The diagnosis and monitoring of CNS lymphoma are still challenging. MRI can be misleading and not sufficiently sensitive, and the difficulties of the intracranial tumor biopsies challenge histological diagnosis. Mutational analysis of the CSF could be useful in these cases, since the presence of MYD88 L265P mutation strongly suggests the diagnosis of PCNSL. ${ }^{13,14,16-18}$ Ours is a proof of concept study aiming to identify and characterize ctDNA in the CSF of patients with CNS lymphoma and to evaluate its role in the management of these patients. We were able to identify ctDNA in the CSF from all patients with restricted CNS disease and show that CSF ctDNA could complement the diagnosis, identify somatic mutations, monitor tumor burden and response to treatment, and facilitate early detection of relapse, as well as detect residual disease after treatment. In addition, our results support the notion that the CSF is a better source of ctDNA than plasma.

Assessment of therapeutic response in CNS lymphomas is usually performed by brain MRI along with FC and cytology. Regrettably, around $50-60 \%$ of patients with CNS lymphoma will eventually relapse. ${ }^{21}$ Notably, we observed that CSF ctDNA was able to detect CNS lesions better than FC and cytology. In addition, CSF ctDNA was able to predict CNS relapse months before MRI or FC, indicating that it can be used for early detection of tumor relapse.

CNS relapse from systemic lymphoma is a fatal complication with an overall survival of less than 6 months, and early identification of patients with CNS relapse is of paramount value. ${ }^{3}$ High-risk patients are usually tested for CNS disease by CSF analysis including cytology and FC. Although FC is a more sensitive test than conventional cytology, ${ }^{5,6}$ it has some limitations ${ }^{22}$ and some patients with no evidence of CNS disease by FC still relapse, suggesting the presence of undetected malignant cells in the CSF. We identified ctDNA in the CSF in the absence of MRI or FC detection earlier than CNS relapse. This indicates that CSF ctDNA could facilitate early detection of CNS relapse in high-risk systemic lymphomas who undergo serial lumbar punctures to administrate prophylactic IT chemotherapy, therefore improving management of these patients.

Moreover, we observed the persistence of high levels of CSF ctDNA in some patients even though FC did not detect tumor cells. This suggested that the detection of a residual disease by CSF ctDNA could be used to determine the type and duration of treatments.

Taken together, and with the limitation of the reduced number of patients analyzed, our findings show that CSF ctDNA can be an important tool to complement standard procedures to evaluate the CNS lymphoma disease status. This technology can be exploited as a 'liquid biopsy' of CNS lymphoma opening a novel way forward for research in circulating biomarkers of CNS lymphoma with an important impact on the future characterization, diagnosis, prognosis, and management of this type of diseases.

\section{Disclosures}

JS is a co-founder of Mosaic Biomedicals and Northern Biologics. JS received grant/research support from Mosaic Biomedicals, Northern Biologics and Roche/Glycart. M. C. has received research funding from Karyopharm, Pharmacyclics, Roche, Arqule and AstraZeneca. Francesc Bosch has received research funding and honoraria from Roche, Celgene, Takeda, AstraZeneca, Novartis, Abbie and Janssen. All remaining authors have declared no conflicts of interest.

\section{Contributions}

\section{$S B, M C, L E, F B$ and JS contributed equally.}

\section{Funding}

This work was supported by research funding from Fundacion Asociación Española contra el Cáncer (AECC) (to JS, MC and PA); FERO (to JS), la Caixa (to JS), BBVA (CAIMI) (to JS), the Instituto de Salud Carlos III, Fondo de Investigaciones Sanitarias (PI16/01278 to JS; PI17/00950 to MC; PI17/00943 to FB) cofinanced by the European Regional Development Fund (ERDF) and Gilead Fellowships (GLD16/00144, GLD18/00047, to FB). MC holds a contract from Ministerio de Ciencia, Innovación y Universidades (RYC-2012-12018). SB received funding from Fundación Alfonso Martin Escudero. LE received funding from the Juan de la Cierva fellowship. We thank CERCA Programme I Generalitat de Catalunya for institutional support. 


\section{References}

1. Yamamoto W, Tomita N, Watanabe R, et al. Central nervous system involvement in diffuse large B-cell lymphoma. Eur J Haematol. 2010;85(1):6-10.

2.WHO classification of tumours of haematopoietic and lymphoid tissues. Revised 4th ed. Lyon, France: IARC; 2017.

3. El-Galaly TC, Cheah CY, Bendtsen MD, et al. Treatment strategies, outcomes and prognostic factors in 291 patients with secondary CNS involvement by diffuse large B-cell lymphoma. Eur J Cancer. 2018;93:57-68.

4. Grommes C, Rubenstein JL, DeAngelis LM, Ferreri AJM, Batchelor TT. Comprehensive approach to diagnosis and treatment of newly diagnosed primary CNS lymphoma. Neuro Oncol. 2019;21(3):296-305.

5. Quijano S, López A, Manuel Sancho J, et al. Identification of leptomeningeal disease in aggressive B-cell non-Hodgkin's lymphoma: improved sensitivity of flow cytometry. J Clin Oncol. 2009;27(9):1462-1469.

6. Hegde U, Filie A, Little RF, et al. High incidence of occult leptomeningeal disease detected by flow cytometry in newly diagnosed aggressive B-cell lymphomas at risk for central nervous system involvement: the role of flow cytometry versus cytology. Blood. 2005;105(2):496-502.

7. Roschewski M, Dunleavy K, Pittaluga S, et al. Circulating tumour DNA and CT monitoring in patients with untreated diffuse large B-cell lymphoma: a correlative biomarker study. Lancet Oncol. 2015;16(5):541-549.

8. Kurtz DM, Scherer F, Jin MC, et al Circulating tumor DNA measurements as early outcome predictors in diffuse large Bcell lymphoma. J Clin Oncol. 2018; 36(28):2845-2853

9. De Mattos-Arruda L, Mayor R, Ng CKY, et al. Cerebrospinal fluid-derived circulating tumour DNA better represents the genomic alterations of brain tumours than plasma. Nat Commun. 2015;6:8839.

10. Pentsova EI, Shah RH, Tang J, et al Evaluating cancer of the central nervous system through next-generation sequencing of cerebrospinal fluid. J Clin Oncol. 2016; 34(20):2404-2415.

11. Momtaz P, Pentsova E, Abdel-Wahab O, et al. Quantification of tumor-derived cell free DNA(cfDNA) by digital PCR (DigPCR) in cerebrospinal fluid of patients with BRAFV600 mutated malignancies. Oncotarget. 2016;7(51):85430-85436.

12. Martínez-Ricarte F, Mayor R, Martínez-Sáez E, et al. Molecular diagnosis of diffuse gliomas through sequencing of cell-free circulating tumor DNA from cerebrospinal fluid. Clin Cancer Res. 2018;24(12):28122819.

13. Fontanilles M, Marguet F, Bohers É, et al. Non-invasive detection of somatic mutations using next-generation sequencing in primary central nervous system lymphoma. Oncotarget. 2017;8(29):48157-48168.

14. Hattori K, Sakata-Yanagimoto M, Suehara Y, et al. Clinical significance of disease-specific MYD88 mutations in circulating DNA in primary central nervous system lymphoma. Cancer Sci. 2018;109(1):225-230.

15. Grommes C, Tang SS, Wolfe J, et al. Phase $1 \mathrm{~b}$ trial of an ibrutinib-based combination therapy in recurrent/refractory CNS lym- phoma. Blood. 2019;133(5):436-445

16. Hiemcke-Jiwa LS, Leguit RJ, Snijders TJ, et al. MYD88 p.(L265P) detection on cell-free DNA in liquid biopsies of patients with primary central nervous system lymphoma. Br J Haematol. 2019;185(5):974-977.

17. Hiemcke-Jiwa LS, Minnema MC, Radersma-van Loon $\mathrm{JH}$, et al. The use of droplet digital PCR in liquid biopsies: a high ly sensitive technique for MYD88 p.(L265P) detection in cerebrospinal fluid. Hemato Oncol. 2018;36(2):429-435.

18. Hickmann AK, Frick M, Hadaschik D, et al Molecular tumor analysis and liquid biopsy: a feasibility investigation analyzing circulating tumor DNA in patients with central nervous system lymphomas. BMC Cancer. 2019;19(1):192.

19. Rimelen V, Ahle G, Pencreach E, et al Tumor cell-free DNA detection in CSF for primary CNS lymphoma diagnosis. Acta Neuropathol Commun. 2019;7(1):43.

20. Forshew T, Murtaza M, Parkinson C et al. Noninvasive identification and monitoring of cancer mutations by targeted deep sequencing of plasma DNA. Sci Transl Med. 2012;4(136):136ra168.

21. Langner-Lemercier S, Houillier C, Soussain C, et al. Primary CNS lymphoma at first relapse/progression: characteristics, management, and outcome of 256 patients from the French LOC network. Neuro Oncol. 2016;18(9):1297-1303.

22. Canovi S, Campioli D. Accuracy of flow cytometry and cytomorphology for the diagnosis of meningeal involvement in lym phoid neoplasms: a systematic review Diagn Cytopathol. 2016;44(10):841-856. 